\title{
Preservation of Sahlqvist fixed point equations in completions of relativized fixed point Boolean algebras with operators
}

\author{
Nick Bezhanishvili AND IAN HodKinson
}

Abstract. We define Sahlqvist fixed point equations and relativized fixed point Boolean algebras with operators (relativized fixed point BAOs). We show that every Sahlqvist fixed point equation is preserved under completions of conjugated relativized fixed point BAOs. This extends the result of Givant and Venema [8] to the setting of relativized fixed point BAOs.

\section{Introduction}

Sahlqvist equations form an important class of equations that are preserved under canonical extensions of Boolean algebras with operators (BAOs for short). In [15] Sambin and Vaccaro gave an order-topological proof of this preservation result, and Jónsson [10] proved it by algebraic means. An essential ingredient of Sambin and Vaccaro's proof is Esakia's lemma of [6], whereas Jónsson's proof relies on a result by Ribeiro [14]. In [4], analogues of Sahlqvist's preservation theorem and of Esakia's lemma were proved for modal fixed point logic. Modal fixed point logic is an extension of standard modal logic with fixed point operators. This formalism is becoming increasingly popular in mainstream computer science applications as it provides a very expressive and yet decidable tool for reasoning. The current paper can be seen as a sequel to [4], where Sahlqvist fixed point formulas are defined and shown to be preserved under canonical extensions of modal fixed point algebras. Here we prove another preservation result for Sahlqvist fixed point equations (which are algebraic analogues of Sahlqvist fixed point formulas), but this time for completions of relativized fixed point BAOs.

Similarly to canonical extensions, completions play an important role in the theory of BAOs. Dedekind used completions of the rational numbers via the so-called Dedekind cuts as a way of introducing the real numbers. MacNeille [12] extended this construction to any poset and showed that any poset can be embedded into a complete lattice. Using the same method, it can be shown that a completion of a Boolean (Heyting) algebra is again a Boolean (Heyting) algebra. But a completion of a distributive lattice may not be a distributive lattice (e.g., [1]). In [13] Monk extended the definition of a completion to BAOs. Nowadays this construction is called the Dedekind-MacNeille completion, MacNeille completion or Monk completion. It will probably be fair to call the completions of BAOs Dedekind-MacNeille-Monk completions 
or MacNeille-Monk completions. However, to avoid confusion we will just call them completions.

Completions of BAOs have been quite well investigated. Monk [13] proved that every positive equation is preserved under completions. Givant and Venema [8] extended this result by showing that all Sahlqvist equations are preserved under completions of conjugated BAOs (see also [18]). Givant and Venema also gave an example of a Sahlqvist equation not preserved under non-conjugated BAOs. Thus, the condition that the BAOs are conjugated cannot be dropped. Gehrke, Harding, Venema [7] showed that every variety of lattices with monotone operators (in particular, BAOs) that is closed under completions is also closed under canonical extensions. In [9] Harding and Bezhanishvili characterized the varieties of modal algebras closed under completions.

We extend the result of Givant and Venema to Sahlqvist fixed point equations and conjugated BAOs with fixed point operators. Our semantics is 'nonclassical'. For each BAO $\mathcal{A}$ we fix a set $\mathcal{F}$ of its elements and evaluate fixed point operators relative to $\mathcal{F}$. In other words, we evaluate the least (greatest) fixed point operator of a given monotone function $f$ as the meet (join) of not all pre-fixed points (post-fixed points) of $f$, but of only those that belong to $\mathcal{F}$. We call the pair $(\mathcal{A}, \mathcal{F})$ a relativized fixed point BAO. Completions of fixed point BAOs for classical semantics were already investigated by Santocanale in [16]. In particular, Santocanale showed inter alia that there exists a conjugated fixed point $\mathrm{BAO}$ that does not admit an embedding into a complete fixed point $\mathrm{BAO}$ preserving fixed point formulas, interpreted in classical semantics. Therefore, fixed point BAOs may not be subalgebras of their completions, whereas relativized fixed point BAOs are subalgebras of their completions. Thus, relativized semantics provides a workable notion of completion for which we can prove positive (preservation) results.

We note that our proof also works for the language without fixed point operators. Thus, we obtain the result of [8] as a corollary of our main proof. Our proof seems to be different from the one of [8] and [18] and is similar to the proofs of [15], [2], and [4], whereas Venema and Givant's approach is closer to the approach of [10]. Our proof, however, is also different (and in a way simpler) than the proofs of [15] and [4], for a few reasons. We work with conjugated BAOs, not arbitrary BAOs. Also the 'minimal' assignment used in our proof is defined in the original algebra. In the topological terminology of [15] and [4], this means that it is a 'clopen', not just a 'closed' assignment. As a result, we do not need to prove an analogue of the Esakia lemma, which is necessary in [15] and [4]. Moreover, unlike [4] (where the analogue of the Esakia lemma is proved only for the language with the least fixed point operator), in this paper we work with the fixed point language with both least and greatest fixed point operators. 
The paper is organized as follows: In Section 2 we introduce (relativized) fixed point BAOs. In Section 3 we recall the main properties of completions of BAOs and define completions for relativized fixed point BAOs. In Section 4 we define Sahlqvist fixed point equations and prove the main theorem of the paper that Sahlqvist fixed point equations are preserved under completions of conjugated fixed point BAOs. Finally, we conclude the paper by listing some future work in Section 5.

Acknowledgment The work of the first author was partially supported by the EPSRC grant EP/F032102/1. The authors are very grateful to John Harding for interesting discussions as well as to the referee and editor for helpful and informative comments.

\section{Relativized fixed point BAOs}

In this section we recall the language of modal $\mu$-calculus and define fixed point BAOs. We fix a countably infinite set $V$ of variables $\left(x, y, x_{0}, x_{1}\right.$, etc) and a set $L$ of operator symbols of any arity. The set of fixed point terms is then defined as the least set such that

- constants $\perp$ and $\top$ and variables are fixed point terms,

- if $t_{1}$ and $t_{2}$ are fixed point terms, then $t_{1} \wedge t_{2}, t_{1} \vee t_{2}, \neg t_{1}$ are fixed point terms,

- for each $n$-ary operator $f \in L$, and fixed point terms $t_{1}, \ldots, t_{n}, f\left(t_{1}, \ldots\right.$, $\left.t_{n}\right)$ is also a fixed point term,

- for each fixed point term $\gamma\left(x, x_{1}, \ldots, x_{n}\right)$, where $x$ occurs under the scope of an even number of negations, $\mu x \gamma\left(x, x_{1}, \ldots, x_{n}\right)$ and $\nu x \gamma\left(x, x_{1}, \ldots, x_{n}\right)$ are fixed point terms.

A fixed point term that does not contain any $\mu$ or $\nu$-operators will be called an $L$-term. If $\gamma=\mu x \delta$ or $\gamma=\nu x \delta^{\prime}$, then we will say that $x$ is a bound variable in $\gamma$. If $x$ does not occur in the scope of any $\mu x$ or $\nu x$, it is called a free variable. We say that a fixed point term $\gamma\left(x, x_{1}, \ldots x_{n}\right)$ is positive in $x$ (negative in $x$ ) if all the free occurrences of the variable $x$ are under the scope of an even (odd) number of negations.

Let $(A, \vee, \wedge, \neg, 0,1)$ be a Boolean algebra. Recall that a map $f: A^{n} \rightarrow A$ is called a normal and additive operator if for each $i \leq n$ and $a, b, a_{1}, \ldots, a_{n} \in A$ we have

(1) $f\left(a_{1}, \ldots, a_{i-1}, 0, a_{i+1}, \ldots, a_{n}\right)=0$,

(2) $f\left(a_{1}, \ldots, a_{i-1}, a \vee b, a_{i+1}, \ldots, a_{n}\right)=f\left(a_{1}, \ldots, a_{i-1}, a, a_{i+1}, \ldots, a_{n}\right)$

$$
\vee f\left(a_{1}, \ldots, a_{i-1}, b, a_{i+1}, \ldots, a_{n}\right) \quad \text { (Additivity) }
$$

Recall also that a Boolean algebra with operators ( $B A O$ for short) is an algebra $\mathcal{A}=\left(A, \vee, \wedge, \neg, 0,1, f^{\mathcal{A}}: f \in L\right)$, where $(A, \vee, \wedge, \neg, 0,1)$ is a Boolean algebra and each $f^{\mathcal{A}}$ is a normal additive operator. If it is clear from the 
context we will skip the index $\mathcal{A}$. We will often use the shorthand $\mathcal{A}=(A, f$ : $f \in L)$ for denoting BAOs. For each $n$-ary operator symbol $f \in L$ and fixed point terms $t_{1}, \ldots, t_{n}$ we let $f^{d}\left(t_{1}, \ldots, t_{n}\right)$ denote the fixed point term $\neg f\left(\neg t_{1}, \ldots, \neg t_{n}\right)$.

\section{DEFINITION 2.1 .}

(1) Let $\mathcal{A}=\left(A, f^{\mathcal{A}}: f \in L\right)$ be a $\mathrm{BAO}$ and $\mathcal{F} \subseteq \mathcal{A}$. A map $h$ from variables to $A$ is called an assignment. We define a (possibly partial) semantics for fixed point terms by the following inductive definition.

$$
\begin{aligned}
& {[\perp]_{h, \mathcal{F}}^{\mathcal{A}}=0 \text { and }[T]_{h, \mathcal{F}}^{\mathcal{A}}=1,} \\
& {[x]_{h, \mathcal{F}}^{\mathcal{A}}=h(x), \text { where } x \text { is a variable, }} \\
& {[\gamma \wedge \delta]_{h, \mathcal{F}}^{\mathcal{A}}=[\gamma]_{h, \mathcal{F}}^{\mathcal{A}} \wedge[\delta]_{h, \mathcal{F}}^{\mathcal{A}},} \\
& {[\gamma \vee \delta]_{h, \mathcal{F}}^{\mathcal{A}}=[\gamma]_{h, \mathcal{F}}^{\mathcal{A}} \vee[\delta]_{h, \mathcal{F}}^{\mathcal{A}},} \\
& {[\neg \gamma]_{h, \mathcal{F}}^{\mathcal{A}}=\neg[\gamma]_{h, \mathcal{F}}^{\mathcal{A}}} \\
& {\left[f\left(\gamma_{1}, \ldots, \gamma_{n}\right)\right]_{h, \mathcal{F}}^{\mathcal{A}}=f^{\mathcal{A}}\left(\left[\gamma_{1}\right]_{h, \mathcal{F}}^{\mathcal{A}}, \ldots,\left[\gamma_{n}\right]_{h, \mathcal{F}}^{\mathcal{A}}\right), \text { for each } n \text {-ary } f \in L .}
\end{aligned}
$$

For $a \in A$ and $x$ a variable, we denote by $h_{x}^{a}$ a new assignment such that $h_{x}^{a}(x)=a$ and $h_{x}^{a}(y)=h(y)$ for each variable $y \neq x$.

If $\gamma\left(x, x_{1}, \ldots, x_{n}\right)$ is positive in $x$, then we let

$$
\left[\mu x \gamma\left(x, x_{1}, \ldots, x_{n}\right)\right]_{h, \mathcal{F}}^{\mathcal{A}}=\bigwedge\left\{a \in \mathcal{F}:\left[\gamma\left(x, x_{1}, \ldots, x_{n}\right)\right]_{h_{x}^{a}, \mathcal{F}}^{\mathcal{A}} \leq a\right\},
$$

if this meet exists; otherwise, the semantics for $\mu x \gamma\left(x, x_{1}, \ldots, x_{n}\right)$ is undefined, and we let

$$
\left[\nu x \gamma\left(x, x_{1}, \ldots, x_{n}\right)\right]_{h, \mathcal{F}}^{\mathcal{A}}=\bigvee\left\{a \in \mathcal{F}:\left[\gamma\left(x, x_{1}, \ldots, x_{n}\right)\right]_{h_{x}^{a}, \mathcal{F}}^{\mathcal{A}} \geq a\right\},
$$

if this join exists; otherwise, the semantics for $\nu x \gamma\left(x, x_{1}, \ldots, x_{n}\right)$ is undefined.

An element $a$ such that $\left[\gamma\left(x, x_{1}, \ldots, x_{n}\right)\right]_{h_{x}^{a}, \mathcal{F}}^{\mathcal{A}} \leq a$ (respectively, $\left[\gamma\left(x, x_{1}\right.\right.$, $\left.\left.\ldots, x_{n}\right)\right]_{h_{x}^{a}, \mathcal{F}}^{\mathcal{A}} \geq a$ ) will be called a pre-fixed point (resp. post-fixed point) of $\gamma$.

(2) A BAO $\mathcal{A}$ is called a fixed point $B A O$ relative to $\mathcal{F} \subseteq \mathcal{A}$ if $[\gamma]_{h}^{\mathcal{F}}$ is defined for any fixed point term $\gamma$ and any assignment $h$. The pair $(\mathcal{A}, \mathcal{F})$ is then called a relativized fixed point $B A O$.

(3) We say that an equation $\gamma=\delta$, where $\gamma$ and $\delta$ are fixed point terms, is valid in a fixed point BAO $\mathcal{A}$ relative to $\mathcal{F} \subseteq \mathcal{A}$ if $[\gamma]_{h}^{\mathcal{F}}=[\delta]_{h}^{\mathcal{F}}$ for each assignment $h$.

Notation: To simplify the notations instead of $\left[\gamma\left(x_{1}, \ldots, x_{n}\right)\right]_{h, \mathcal{F}}^{\mathcal{A}}$ with $h\left(x_{i}\right)=$ $a_{i}, 1 \leq i \leq n$, we will write $\gamma\left(a_{1}, \ldots, a_{n}\right)_{\mathcal{F}}^{\mathcal{A}}$. We will also skip the indexes $\mathcal{A}$ and $\mathcal{F}$ if they are clear from the context.

Examples: Every $\mathrm{BAO} \mathcal{A}$ is a fixed point $\mathrm{BAO}$ relative to any finite $\mathcal{F} \subseteq \mathcal{A}$. Recall that a $\mathrm{BAO} \mathcal{A}=(A, f: f \in L)$ is called complete if $A$ is a complete Boolean algebra; that is, for each subset $S$ of $A$ the meet $\bigwedge S$ and the join $\bigvee S$ 
exist. It is straightforward to see that every complete $\mathrm{BAO} \mathcal{A}$ is a fixed point $\mathrm{BAO}$ relative to any $\mathcal{F} \subseteq \mathcal{A}$. Locally finite BAOs are other examples of fixed point BAOs (see [4] for details).

LEMMA 2.2. Let $\mathcal{A}$ be a fixed point $B A O$ relative to $\mathcal{F} \subseteq \mathcal{A}$, and $a_{1}, \ldots$, $a_{n} \in \mathcal{A}$. Then for each fixed point term $\gamma\left(x, x_{1} \ldots, x_{n}\right)$ that is positive in $x$, $\gamma\left(\cdot, a_{1}, \ldots, a_{n}\right)_{\mathcal{F}}^{\mathcal{A}}$ is a monotone function. That is, for $a, b \in \mathcal{A}$,

$$
a \leq b \text { implies } \gamma\left(a, a_{1}, \ldots, a_{n}\right)_{\mathcal{F}}^{\mathcal{A}} \leq \gamma\left(b, a_{1}, \ldots, a_{n}\right)_{\mathcal{F}}^{\mathcal{A}}
$$

Proof. We will prove the lemma by induction on the complexity of $\gamma$. We will skip the indexes $\mathcal{A}$ and $\mathcal{F}$. Our induction hypothesis is: 1$)$ if $\gamma\left(x, x_{1} \ldots, x_{n}\right)$ is positive in $x$, then $\gamma\left(\cdot, a_{1}, \ldots, a_{n}\right)$ is monotone and 2) if $\gamma\left(x, x_{1} \ldots, x_{n}\right)$ is negative in $x$, then $\gamma\left(\cdot, a_{1}, \ldots, a_{n}\right)$ is antitone. The cases $\gamma=\perp, \gamma=\top, \gamma$ is a variable, $\gamma=\delta \vee \delta^{\prime}, \gamma=\delta \wedge \delta^{\prime}, \gamma=\neg \delta$ and $\gamma=f\left(\delta_{1}, \ldots, \delta_{n}\right)$ are proved as in standard modal logic (see, e.g., $[5,8]$ ). Now let $\gamma=\mu y \delta\left(y, x, x_{1}, \ldots, x_{n}\right)$ be positive in $x$ and inductively assume the result for $\delta$. Then, by the induction hypothesis, for each $a, b \in \mathcal{A}$ with $a \leq b$ and $c \in \mathcal{A}$ we have $\delta\left(c, a, a_{1}, \ldots\right.$, $\left.a_{n}\right) \leq \delta\left(c, b, a_{1}, \ldots, a_{n}\right)$. So if $\delta\left(c, b, a_{1}, \ldots, a_{n}\right) \leq c$, then $\delta\left(c, a, a_{1}, \ldots, a_{n}\right) \leq$ $c$. Therefore, the set $\left\{c \in \mathcal{F}: \gamma\left(c, a, a_{1}, \ldots, a_{n}\right) \leq c\right\}$ contains the set $\{c \in \mathcal{F}$ : $\left.\gamma\left(c, b, a_{1}, \ldots, a_{n}\right) \leq c\right\}$. But this means that $\mu y \delta\left(y, a, a_{1}, \ldots, a_{n}\right)=\bigwedge\{c \in \mathcal{F}$ : $\left.\delta\left(c, a, a_{1}, \ldots, a_{n}\right) \leq c\right\} \leq \bigwedge\left\{c \in \mathcal{F}: \delta\left(c, b, a_{1}, \ldots, a_{n}\right) \leq c\right\}=\mu y \delta\left(y, b, a_{1}, \ldots\right.$, $\left.a_{n}\right)$. Therefore, we obtained that $\gamma\left(a, a_{1}, \ldots, a_{n}\right) \leq \gamma\left(b, a_{1}, \ldots, a_{n}\right)$. The cases of $\delta$ negative in $x$ and $\gamma=\nu y \delta\left(y, x, x_{1}, \ldots, x_{n}\right)$ are similar.

\section{Completions and conjugates}

In this section we recall the notion of a completion for BAOs and extend the definition of a completion to fixed point BAOs. We also discuss conjugates of functions.

Let $A$ and $B$ be Boolean algebras such that $A$ is a subalgebra of $B$. We say that $A$ is a dense subalgebra of $B$ if for each $0<b \in B$ there exists $0<a \in A$ such that $a \leq b$. It is easy to see that if $A$ is a dense subalgebra of $B$ then for each $b \in B$ we have $b=\bigvee^{B}\{a \in A: 0<a \leq b\}$.

The MacNeille completion $\bar{A}$ of a Boolean algebra $A$ is a complete Boolean algebra $\bar{A}$ such that $A$ is a dense subalgebra of $\bar{A}$ and all the joins and meets that exist in $A$ are preserved in $\bar{A}$. In other words, if $S \subseteq A$ is such that $\bigvee^{A} S$ exists, then $\bigvee^{A} S=\bigvee^{\bar{A}} S$ and if $T \subseteq A$ is such that $\bigwedge^{A} T$ exists, then $\bigwedge^{A} T=\bigwedge^{\bar{A}} T$. The completion of a Boolean algebra always exists and is unique up to isomorphism (see, e.g., [17]). We assume throughout that the embedding of $A$ into its MacNeille completion is the identity.

An $n$-ary map $f$ on a Boolean algebra $A$ is called completely additive in the $i$-th coordinate if for each (possibly empty) $S \subseteq \mathcal{A}$ such that $\bigvee S$ exists in $\mathcal{A}$ and $a_{1}, \ldots, a_{n} \in \mathcal{A}$ we have

$f\left(a_{1}, \ldots, a_{i-1}, \bigvee S, a_{i+1}, \ldots, a_{n}\right)=\bigvee\left\{f\left(a_{1}, \ldots, a_{i-1}, s, a_{i+1}, \ldots, a_{n}\right): s \in S\right\}$. 
An $n$-ary operator $f$ is called completely additive if it is completely additive in the $i$-th coordinate for each $i \leq n$. A BAO $\mathcal{A}=(A, f: f \in L)$ is called completely additive if each $f \in L$ is completely additive.

Let $\mathcal{A}=(A, f: f \in L)$ be a completely additive BAO. Then for each $f \in L$ the (lower) extension of $f$ to the MacNeille completion $\bar{A}$ of $A$ is defined as:

$$
\bar{f}(b)=\bigvee^{\bar{A}}\{f(a): a \in A, a \leq b\} .
$$

The algebra $\overline{\mathcal{A}}=(\bar{A}, \bar{f}: f \in L)$ is called the completion of $\mathcal{A}=(A, f: f \in L)$.

The next two lemmas summarize the important properties of the completions of BAOs that will be used subsequently. We skip most of the proofs, but give references where they could be found.

LEMMA 3.1. Let $\mathcal{A}=(A, f: f \in L)$ be a completely additive BAO. Then

(1) $\overline{\mathcal{A}}=(\bar{A}, \bar{f}: f \in L)$ is a completely additive BAO.

(2) $\mathcal{A}$ as a $B A O$ is a subalgebra of $\overline{\mathcal{A}}$.

Proof. For the proof of (1) we refer to [13, Theorem 1.2] (see also [8, Lemma 13]). (2) follows from [17, Section 35] and the definition of $\bar{f}$ for each $f \in L$.

Note that an analogue of Lemma 3.1 for fixed point BAOs in classical semantics does not hold [16]. However, we will see next that for relativized fixed point BAOs, we can prove an analogue of Lemma 3.1. Let $(\mathcal{A}, \mathcal{F})$ and $\left(\mathcal{A}^{\prime}, \mathcal{F}^{\prime}\right)$ be relativized fixed point BAOs. We say that $(\mathcal{A}, \mathcal{F})$ is a relativized fixed point subalgebra (or just a subalgebra, for short) of $\left(\mathcal{A}^{\prime}, \mathcal{F}^{\prime}\right)$ if $\mathcal{A}$ is a subalgebra of $\mathcal{A}^{\prime}$ in the BAO sense, and for any assignment $h$ in $\mathcal{A}$ and any term $\gamma$ we have $[\gamma]_{h, \mathcal{F}}^{\mathcal{A}}=[\gamma]_{h, \mathcal{F}^{\prime}}^{\mathcal{A}^{\prime}}$.

LEMMA 3.2. Let $\mathcal{A}=(A, f: f \in L)$ be a completely additive fixed point $B A O$ relative to a set $\mathcal{F} \subseteq \mathcal{A}$. Let also $\overline{\mathcal{A}}=(\bar{A}, \bar{f}: f \in L)$ be the completion of $\mathcal{A}$. Then

(1) $(\overline{\mathcal{A}}, \mathcal{F})$ is a relativized fixed point $B A O$.

$(2)(\mathcal{A}, \mathcal{F})$ is a relativized fixed point subalgebra of $(\overline{\mathcal{A}}, \mathcal{F})$.

Proof. (1) All we need to check is that for each $\gamma\left(x, x_{1}, \ldots, x_{n}\right)$ positive in $x$ and each assignment $g$ in $\bar{A}$, the meet $\bigwedge\left\{a \in \mathcal{F}:\left[\gamma\left(x, x_{1}, \ldots, x_{n}\right)\right]_{g_{x}^{a}} \leq a\right\}$ and join $\bigvee\left\{a \in \mathcal{F}:\left[\gamma\left(x, x_{1}, \ldots, x_{n}\right)\right]_{g_{x}^{a}} \geq a\right\}$ exist in $\bar{A}$. But this is obvious as $\bar{A}$ is a complete BAO.

(2) Let $h$ be any assignment in $A$. We prove by induction on the complexity of each term $\gamma$ that $[\gamma]_{h, \mathcal{F}}^{\mathcal{A}}=[\gamma]_{h, \mathcal{F}}^{\overline{\mathcal{A}}}$. By Lemma 3.1(2), we only need to consider the cases $\gamma=\mu x \delta$ and $\gamma=\nu x \delta$. Let $\gamma=\mu x \delta$. Then

$$
\begin{aligned}
{[\gamma]_{h, \mathcal{F}}^{\mathcal{A}} } & =\bigwedge^{A}\left\{a \in \mathcal{F}:[\delta]_{h_{x}^{a}, \mathcal{F}}^{\mathcal{A}} \leq a\right\} \\
& =\bigwedge^{A}\left\{a \in \mathcal{F}:[\delta]_{h_{x}^{a}, \mathcal{F}}^{\mathcal{A}} \leq a\right\} \\
& =\bigwedge^{\mathcal{A}}\left\{a \in \mathcal{F}:[\delta]_{h_{x}^{a}, \mathcal{F}}^{\mathcal{A}} \leq a\right\} \\
& =[\mu x \delta]_{h, \mathcal{F}}^{\overline{\mathcal{A}}} \\
& =[\gamma]_{h, \mathcal{F}}^{\mathcal{A}} .
\end{aligned}
$$


The first equation above is spelling out the definitions, the second follows from the induction hypothesis, the third from the fact that completions preserve existing meets, and the fourth and fifth are again spelling out of the definitions. The case $\gamma=\nu x \delta$ is similar. Therefore, $(\mathcal{A}, \mathcal{F})$ is a relativized fixed point subalgebra of $(\overline{\mathcal{A}}, \mathcal{F})$.

DEFINITION 3.3. Let $\mathcal{A}=(A, f: f \in L)$ be a completely additive BAO that is a fixed point BAO relative to a set $\mathcal{F} \subseteq \mathcal{A}$. Let also $\overline{\mathcal{A}}=(\bar{A}, \bar{f}: f \in L)$ be the completion of $\mathcal{A}$. We call the relativized fixed point $\operatorname{BAO}(\overline{\mathcal{A}}, \mathcal{F})$ the completion of $(\mathcal{A}, \mathcal{F})$.

Let $A$ be a Boolean algebra. For $1 \leq i \leq n$, a map $f_{i}^{-1}: A^{n} \rightarrow A$ is called the $i$-th conjugate of a map $f: A^{n} \rightarrow A$ if for all $a, b_{1}, \ldots, b_{n} \in A$ we have $a \wedge f\left(b_{1}, \ldots, b_{n}\right)=0$ iff $b_{i} \wedge f_{i}^{-1}\left(b_{1}, \ldots, b_{i-1}, a, b_{i+1}, \ldots, b_{n}\right)=0$. We say that $f$ is conjugated if it has an $i$-th conjugate for each $i \leq n$. A BAO $\mathcal{A}$ is called conjugated if each operator of $\mathcal{A}$ is conjugated. Conjugates are closely related to residuals (see Claim (1) of Theorem 4.7).

\section{LEMMA 3.4.}

(1) Any conjugated $n$-ary map $f$ on a Boolean algebra $A$ is completely additive.

(2) If $\mathcal{A}=(A, f: f \in L)$ is a conjugated BAO, then $\overline{\mathcal{A}}=(\bar{A}, \bar{f}: f \in L)$ is also a conjugated $B A O$.

(3) If $\mathcal{A}$ is a conjugated fixed point $B A O$ relative to some set $\mathcal{F} \subseteq \mathcal{A}$, then $(\overline{\mathcal{A}}, \mathcal{F})$ is also a conjugated fixed point $B A O$ relative to $\mathcal{F}$.

Proof. For the proof of (1) we refer to [11, Theorem 1.14] (see also [8, Lemma 19]). For (2), we first observe that by (1), $\mathcal{A}=(A, f: f \in L)$ is completely additive and so the completion $\overline{\mathcal{A}}=(\bar{A}, \bar{f}: f \in L)$ exists. The result now follows from the fact that if $f_{i}^{-1}$ is the $i$-th conjugate of $f$ on $A$, then $\overline{f_{i}^{-1}}$ is the $i$-th conjugate of $\bar{f}$ on $\bar{A}$ (see [13, Corollary 1.11] and also [8, Lemma 20]). (3) follows from (1), (2) and Lemma 3.2(1).

\section{Main results}

In this section we define Sahlqvist fixed point terms and equations and prove the main theorem of this paper that every Sahlqvist fixed point equation is preserved under completions of conjugated relativized fixed point BAOs.

DEFINITION 4.1. A fixed point term $\gamma$ is called positive if it is positive in each variable, and $\gamma$ is called negative if $\neg \gamma$ is positive.

\section{DEFINITION 4.2.}

(1) A skeleton is a term $\alpha\left(x_{1}, \ldots, x_{n}\right)$ made from operators and $\wedge$ only, in which no variable occurs twice. 
(2) A Sahlqvist fixed point term is a fixed point term of the form $\alpha\left(\gamma_{i} / x_{i}: i=\right.$ $1, \ldots, n)$, where $\alpha\left(x_{1}, \ldots, x_{n}\right)$ is a skeleton and the $\gamma_{i}$ are either negative fixed point terms, or 'boxed variables' of the form $f_{1}^{d} \ldots f_{k}^{d} y$ for $k<\omega$ and unary $f_{1}, \ldots, f_{k} \in L$.

(3) A Sahlqvist fixed point equation is one of the form $\gamma=0$ where $\gamma$ is a Sahlqvist fixed point term.

REMARK 4.3. If we drop the fixed point operators, this definition is essentially the same as a standard one from [5], when translated from modal to algebraic notation. As notation, we let 'boxed-variables' denote the set of all terms of the form $f_{1}^{d} \ldots f_{k}^{d} y$ as above, 'positive' the set of positive $L$ terms, and 'negative' the set of negative $L$-terms. For sets $S, T$ of $L$-terms and a set $F$ of function symbols, we let $S^{F}$ denote the closure of $S$ under the symbols in $F, \neg S=\{\neg \gamma: \gamma \in S\}, S \wedge T=\{\gamma \wedge \delta: \gamma \in S, \delta \in T\}$, and $S \rightarrow T=\{\neg \gamma \vee \delta: \gamma \in S, \delta \in T\}$. Let $\oplus, \otimes$ denote disjunction and conjunction $(\vee, \wedge)$, respectively, over terms with no variables in common, and write $L^{d}=\left\{f^{d}: f \in L\right\}$. Finally, let $S$ be the set of $L$-terms that are Sahlqvist fixed point terms, as defined above. Now, phrased algebraically, [5, Definition 3.51] defines a Sahlqvist formula (in the language without fixed point operators) as a term in the set

$$
\Sigma=\left((\{\top, \perp\} \cup \text { boxed-variables } \cup \text { negative })^{L \cup\{\wedge, \vee\}} \rightarrow \text { positive }\right)^{L^{d} \cup\{\wedge, \oplus\}} .
$$

Clearly, up to logical equivalence we have

$$
\begin{aligned}
\neg \Sigma & =\left((\{\top, \perp\} \cup \text { boxed-variables } \cup \text { negative })^{L \cup\{\wedge, \vee\}} \wedge \text { negative }\right)^{L \cup\{\vee, \otimes\}} \\
& =\left((\text { boxed-variables } \cup \text { negative })^{L \cup\{\wedge, \vee\}}\right)^{L \cup\{\vee, \otimes\}} \\
& =(\text { boxed-variables } \cup \text { negative })^{L \cup\{\wedge, \vee\}} \\
& =\left((\text { boxed-variables } \cup \text { negative })^{L \cup\{\wedge\}}\right)^{\{\vee\}} \\
& =S^{\{\vee\}} .
\end{aligned}
$$

It follows that any Sahlqvist formula $\gamma$ in the sense of [5, Definition 3.51] is equivalent to a conjunction $\bigwedge_{i} \neg \delta_{i}$ where the $\delta_{i}$ are Sahlqvist fixed point terms as above (viewed as $L$-terms), and vice versa. Plainly, $\gamma$ being valid corresponds to $\bigwedge_{i} \neg \delta_{i}=1$ being valid: that is, $\bigwedge_{i}\left(\delta_{i}=0\right)$, a conjunction of ( $L$-equations that are) Sahlqvist fixed point equations as in Definition 4.2.

Also note that Sahlqvist fixed point equations are algebraic analogues of Sahlqvist fixed point formulas of [4].

DEFINITION 4.4. Let $\mathcal{A}$ be a BAO. An L-term $\alpha\left(x_{1}, \ldots, x_{n}\right)$ is called conjugated in $\mathcal{A}$ if it defines a conjugated function in $\mathcal{A}$.

Next we prove two auxiliary lemmas that will play an important role in the proof of the main result.

LEMMA 4.5. If $\mathcal{A}$ is a conjugated BAO, then every skeleton is conjugated in $\mathcal{A}$. 
Proof. We prove the result by induction on the complexity of $\alpha$. The base case is when $\alpha$ is a variable and is trivial. We remark that $\wedge$ can be treated as a binary operator since it is self-conjugated, normal, and additive. Let $f$ be an $n$-ary conjugated operator and let $\alpha_{1}\left(x_{11}, \ldots, x_{1 k_{1}}\right), \ldots, \alpha_{n}\left(x_{n 1}, \ldots, x_{n k_{n}}\right)$ be terms written with $\wedge$ and operators only such that $f\left(\alpha_{1}, \ldots, \alpha_{n}\right)=\alpha$ and no variable occurs twice in $\alpha$. Assume the lemma for all skeletons simpler than $\alpha$. We show that $\alpha$ is a conjugated term. Let $h$ be an assignment on $\mathcal{A}$. Let also $a \in \mathcal{A}$ and fix a variable $x_{i j}$. Then obviously,

$$
a \wedge[\alpha]_{h}=0 \text { iff } a \wedge f\left(\left[\alpha_{1}\right]_{h}, \ldots,\left[\alpha_{n}\right]_{h}\right)=0 .
$$

As $f$ is conjugated, there exists a function $f_{i}^{-1}$ such that (2) holds iff

$$
\left[\alpha_{i}\right]_{h} \wedge f_{i}^{-1}\left(\left[\alpha_{1}\right]_{h}, \ldots,\left[\alpha_{i-1}\right]_{h}, a,\left[\alpha_{i+1}\right]_{h}, \ldots,\left[\alpha_{n}\right]_{h}\right)=0 .
$$

Note that the complexity of each $\alpha_{i}$ is smaller than that of $\alpha$ and no variable occurs twice in any of $\alpha_{i}$. Therefore, by the induction hypothesis, $\alpha_{i}$ is conjugated. So there exists a function $s_{i j}$ such that (3) holds iff

$$
\begin{array}{r}
s_{i j}\left(h\left(x_{i 1}\right), \ldots, h\left(x_{i j-1}\right), f_{i}^{-1}\left(\left[\alpha_{1}\right]_{h}, \ldots,\left[\alpha_{i-1}\right]_{h}, a,\left[\alpha_{i+1}\right]_{h}, \ldots,\left[\alpha_{n}\right]_{h}\right),\right. \\
\left.h\left(x_{i j+1}\right), \ldots, h\left(x_{i k_{i}}\right)\right) \wedge h\left(x_{i j}\right)=0 .
\end{array}
$$

As no variable occurs twice in $\alpha$, this means that $\alpha$ is conjugated.

\section{LEMMA 4.6.}

(1) Let $\mathcal{A}$ and $\mathcal{B}$ be BAOs such that $\mathcal{A}$ is a dense subalgebra of $\mathcal{B}$. Let also $\alpha\left(x_{1}, \ldots, x_{p}, y_{1}, \ldots, y_{q}\right)$ be a conjugated term in $\mathcal{B}$, and $b_{1}, \ldots, b_{p}, n_{1}$, $\ldots, n_{q} \in \mathcal{B}$ be such that $\alpha\left(b_{1}, \ldots, b_{p}, n_{1}, \ldots, n_{q}\right)>0$. Then there are $a_{1}$, $\ldots, a_{p} \in \mathcal{A}$ with $0<a_{i} \leq b_{i}$ (for each $i \leq p$ ) and $\alpha\left(a_{1}, \ldots, a_{p}, n_{1}, \ldots\right.$, $\left.n_{q}\right)>0$.

(2) Let $\mathcal{A}$ be a completely additive $B A O$ and $\overline{\mathcal{A}}$ its completion. Let also $\alpha\left(x_{1}, \ldots, x_{p}, y_{1}, \ldots, y_{q}\right)$ be a conjugated term in $\overline{\mathcal{A}}$, and let $b_{1}, \ldots, b_{p}$, $n_{1}, \ldots, n_{q} \in \overline{\mathcal{A}}$ be such that $\alpha\left(b_{1}, \ldots, b_{p}, n_{1}, \ldots, n_{q}\right)>0$. Then there are $a_{1}, \ldots, a_{p} \in \mathcal{A}$ with $0<a_{i} \leq b_{i}($ for each $i \leq p)$ and $\alpha\left(a_{1}, \ldots, a_{p}, n_{1}, \ldots\right.$, $\left.n_{q}\right)>0$.

Proof. (1) By induction on $p$. If $p=0$, there is nothing to prove. Assume the result for $p$. We prove it it for $p+1$. As $\mathcal{A}$ is dense in $\mathcal{B}, b_{p+1}=\bigvee^{\mathcal{B}}\{a \in \mathcal{A}$ : $\left.0<a \leq b_{p+1}\right\}$. Since $\alpha$ is conjugated in $\mathcal{B}$, by Lemma 3.4(1) it is completely additive in $\mathcal{B}$, so

$$
\begin{gathered}
0<\alpha\left(b_{1}, \ldots, b_{p+1}, n_{1}, \ldots, n_{q}\right)=\bigvee^{\mathcal{B}}\left\{\alpha \left(b_{1}, \ldots, b_{p}, a, n_{1}, \ldots,\right.\right. \\
\left.\left.n_{q}\right): a \in \mathcal{A}, 0<a \leq b_{p+1}\right\} .
\end{gathered}
$$

It follows that $\alpha\left(b_{1}, \ldots, b_{p}, a, n_{1}, \ldots, n_{q}\right)>0$ for some $a \in \mathcal{A}$ with $0<a \leq$ $b_{p+1}$. The result now follows by the inductive hypothesis. (2) follows from (1) and the fact that each $\mathrm{BAO}$ is a dense subalgebra of its completion.

THEOREM 4.7. (Main Theorem) 
(1) Let $\mathcal{A}$ be a conjugated fixed point $B A O$ relative to $\mathcal{F} \subseteq \mathcal{A}$, and let $(\overline{\mathcal{A}}, \mathcal{F})$ be the completion of $(\mathcal{A}, \mathcal{F})$. Then any Sahlqvist fixed point equation valid in $\mathcal{A}$ relative to $\mathcal{F}$ is also valid in $\overline{\mathcal{A}}$ relative to $\mathcal{F}$.

(2) (Givant and Venema [8]) Let $\mathcal{A}$ be a conjugated BAO and $\overline{\mathcal{A}}$ its completion. Then any Sahlqvist equation valid in $\mathcal{A}$ is valid in $\overline{\mathcal{A}}$.

Proof. (1) Throughout the proof we will assume that all fixed point operators are interpreted relative to $\mathcal{F}$. So we drop $\mathcal{F}$ as an index. Let $\alpha\left(\beta_{1}, \ldots, \beta_{p}, \gamma_{1}\right.$, $\left.\ldots, \gamma_{q}\right)$ be a Sahlqvist fixed point term, where $\alpha\left(x_{1}, \ldots, x_{p}, y_{1}, \ldots, y_{q}\right)$ is a skeleton, the $\beta_{i}$ are of the form $f_{1}^{d} \ldots f_{k}^{d} y$, and the $\gamma_{j}$ are negative fixed point terms. Suppose $\alpha\left(\beta_{1}, \ldots, \beta_{p}, \gamma_{1}, \ldots, \gamma_{q}\right)=0$ fails in $\overline{\mathcal{A}}$. We show that $\alpha\left(\beta_{1}\right.$, $\left.\ldots, \beta_{p}, \gamma_{1}, \ldots, \gamma_{q}\right)=0$ also fails in $\mathcal{A}$.

That $\alpha\left(\beta_{1}, \ldots, \beta_{p}, \gamma_{1}, \ldots, \gamma_{q}\right)=0$ fails in $\overline{\mathcal{A}}$ means there is an assignment $h$ in $\overline{\mathcal{A}}$ such that $\left[\alpha\left(\beta_{1}, \ldots, \beta_{p}, \gamma_{1}, \ldots, \gamma_{q}\right)\right]_{h}^{\overline{\mathcal{A}}}>0$. Let $b_{i}=\left[\beta_{i}\right]_{h}^{\overline{\mathcal{A}}} \in \overline{\mathcal{A}}$ and $n_{j}=\left[\gamma_{j}\right]_{h}^{\overline{\mathcal{A}}} \in \overline{\mathcal{A}}$ (for each $i \leq p$ and $j \leq q$ ). So $\alpha\left(b_{1}, \ldots, b_{p}, n_{1}, \ldots, n_{q}\right)>0$ in $\overline{\mathcal{A}}$. By Lemma 3.4(2), $\overline{\mathcal{A}}$ is conjugated. So by Lemma $4.5, \alpha\left(x_{1}, \ldots, x_{p}, y_{1}, \ldots\right.$, $\left.y_{q}\right)$ is conjugated in $\overline{\mathcal{A}}$. Hence, by Lemma $4.6(2)$, there exist $a_{1}, \ldots, a_{p} \in \mathcal{A}$ with $0<a_{i} \leq b_{i}$ (for each $\left.i \leq p\right)$ such that $\alpha\left(a_{1}, \ldots, a_{p}, n_{1}, \ldots, n_{q}\right)>0$ in $\overline{\mathcal{A}}$.

Let $g$ be the assignment into $\mathcal{A}$, which is called a 'minimal assignment', given by

$$
g(x)=\bigvee\left\{f_{k}^{-1} \ldots f_{1}^{-1}\left(a_{i}\right): i \leq p, \beta_{i}=f_{1}^{d} \ldots f_{k}^{d} x\right\}
$$

for each variable $x$, where $f^{-1}$ is the conjugate of $f$ in $\mathcal{A}$, for each $f \in L$. Note that if $x$ does not occur in any $\beta_{i}$, then, according to this definition, $g(x)=\bigvee \emptyset=0$.

\section{Claim.}

(i) $\left[\beta_{i}\right]_{g}^{\mathcal{A}} \geq a_{i}$ for each $i \leq p$.

(ii) $g(x) \leq h(x)$ for each variable $x$.

(iii) $\left[\gamma_{j}\right]_{g}^{\mathcal{A}} \geq n_{j}$ for each $j \leq q$.

Proof. (i) First note that for each $a \in \mathcal{A}$ and each unary conjugated operator $f$ we have

$$
a \leq f^{d} f^{-1} a .
$$

That is to say that $f^{d}$ is the right residual of $f$. To see this, we just need to note that $\neg f^{-1}(a) \wedge f^{-1}(a)=0$ iff $a \wedge f \neg f^{-1}(a)=0$ iff $a \leq \neg f \neg f^{-1}(a)=f^{d} f^{-1}(a)$. Now we will prove by induction that

$$
a \leq f_{1}^{d} \ldots f_{k}^{d} f_{k}^{-1} \ldots f_{1}^{-1}(a) .
$$

The basic step follows from (6). Now assume (7) holds for $k-1$ and we prove it for $k$. By (6) we have $f_{k}^{d} f_{k}^{-1} f_{k-1}^{-1} \ldots f_{1}^{-1}(a) \geq f_{k-1}^{-1} \ldots f_{1}^{-1}(a)$. By the fact that each $f_{i}^{d}$ is monotone and using the induction hypothesis we obtain

$$
\begin{aligned}
f_{1}^{d} \ldots f_{k-1}^{d} f_{k}^{d} f_{k}^{-1} \ldots f_{1}^{-1}(a) & \geq f_{1}^{d} \ldots f_{k-1}^{d} f_{k-1}^{-1} \ldots f_{1}^{-1}(a) \\
& \geq a .
\end{aligned}
$$


Now let $\beta_{i}=f_{1}^{d} \ldots f_{k}^{d} x$. Then $\left[\beta_{i}\right]_{g}^{\mathcal{A}}=f_{1}^{d} \ldots f_{k}^{d} g(x) \geq f_{1}^{d} \ldots f_{k}^{d}\left(f_{k}^{-1} \ldots\right.$ $\left.f_{1}^{-1}\left(a_{i}\right)\right) \geq a_{i}$.

(ii) Let $i \leq p$ and assume $\beta_{i}=f_{1}^{d} \ldots f_{k}^{d} x$. As $a_{i} \leq b_{i}$ we have $a_{i} \leq$ $\left[\beta_{i}\right]_{h}^{\overline{\mathcal{A}}}=\left[f_{1}^{d} \ldots f_{k}^{d} x\right]_{h}^{\overline{\mathcal{A}}}=f_{1}^{d} \ldots f_{k}^{d} h(x)$. By conjugation, this implies that $f_{k}^{-1} \ldots f_{1}^{-1}\left(a_{i}\right) \leq h(x)$ in $\overline{\mathcal{A}}$. This holds for each $a_{i}$ and $\beta_{i}$ with $\beta_{i}$ of the form $f_{1}^{d} \ldots f_{k}^{d} x$. So

$$
g(x)=\bigvee\left\{f_{k}^{-1} \ldots f_{1}^{-1}\left(a_{i}\right): i \leq p, \beta_{i}=f_{1}^{d} \ldots f_{k}^{d} x\right\} \leq h(x) .
$$

(iii) As each $\gamma_{j}$ is a negative term for each $j \leq q$, from (2) of this claim and Lemma 2.2, we have $\left[\gamma_{j}\right]_{g}^{\overline{\mathcal{A}}} \geq\left[\gamma_{j}\right]_{h}^{\overline{\mathcal{A}}}=n_{j}$. Since $g$ is an assignment in $\mathcal{A}$, it follows from Lemma 3.2(2) that $\left[\gamma_{j}\right]_{g}^{\mathcal{A}}=\left[\gamma_{j}\right]_{g}^{\overline{\mathcal{A}}}$ for each $j \leq q$. Therefore, we have $\left[\gamma_{j}\right]_{g}^{\mathcal{A}} \geq n_{j}$ for each $j \leq q$.

Recall that $\alpha$ is positive and has no fixed points. Then putting everything together we obtain:

$$
\begin{aligned}
{\left[\alpha\left(\beta_{1}, \ldots, \beta_{p}, \gamma_{1}, \ldots, \gamma_{q}\right)\right]_{g}^{\mathcal{A}} } & =\alpha\left(\left[\beta_{1}\right]_{g}^{\mathcal{A}}, \ldots,\left[\beta_{p}\right]_{g}^{\mathcal{A}},\left[\gamma_{1}\right]_{g}^{\mathcal{A}}, \ldots,\left[\gamma_{q}\right]_{g}^{\mathcal{A}}\right)^{\mathcal{A}} \\
& =\alpha\left(\left[\beta_{1}\right]_{g}^{\mathcal{A}}, \ldots,\left[\beta_{p}\right]_{g}^{\mathcal{A}},\left[\gamma_{1}\right]_{g}^{\mathcal{A}}, \ldots,\left[\gamma_{q}\right]_{g}^{\mathcal{A}}\right)^{\mathcal{A}} \\
& \geq \alpha\left(a_{1}, \ldots, a_{p}, n_{1}, \ldots, n_{q}\right)^{\mathcal{A}} \\
& >0 .
\end{aligned}
$$

So $\alpha\left(\beta_{1}, \ldots, \beta_{p}, \gamma_{1}, \ldots, \gamma_{q}\right)=0$ fails in $\mathcal{A}$, as required.

(2) The proof is the same as the proof of (1) except that we start with any conjugated $\mathrm{BAO}$ (not necessarily a fixed point $\mathrm{BAO}$ ) and we assume that each term is just an $L$-term (not a fixed point term - thus $\mathcal{F}$ plays no role here).

REMARK 4.8. We note again that the 'minimal' assignment $g$ defined in (5) of the proof of Theorem 4.7 is in the original algebra $\mathcal{A}$. In the topological terminology of [15] and [4] this means that $g$ corresponds to a 'clopen', not just a 'closed' assignment. Esakia's lemma and its fixed point analogue are essentially used in the preservation proofs of [15] and [4] to move from a 'closed' assignment to a 'clopen' assignment. As the assignment $g$ in our case is already 'clopen' we do not need to use the aforementioned lemmas. This also enables us, unlike [4] where the analogue of the Esakia lemma is proved only for the language with the least fixed point operator, to work with the fixed point language with both least and greatest fixed point operators.

A natural question to ask is whether in Theorem 4.7(1) we can drop the condition that fixed point operators in $\mathcal{A}$ and $\overline{\mathcal{A}}$ are computed relative to $\mathcal{F}$. One could ask whether the following is true: if the Sahlqvist fixed point equation $\gamma=0$ is valid in $\mathcal{A}$ relative to $\mathcal{A}$, then $\gamma=0$ is valid in $\overline{\mathcal{A}}$ relative to $\overline{\mathcal{A}}$. In other words, can we prove an analogue of Theorem 4.7(1) if we compute fixed point operators in a standard way, as meets (joins) of all pre-fixed points (post-fixed points)? We finish this section by constructing an example of a 
conjugated $\mathrm{BAO}$ (not a fixed point $\mathrm{BAO}$ ) $\mathcal{A}$ and a Sahlqvist fixed point term $\gamma$ such that, with the standard interpretation of fixed point operators, $\gamma=0$ is valid in $\mathcal{A}$ but not in $\overline{\mathcal{A}}$. We leave it as an open problem to find a fixed point $\mathrm{BAO}$ and a Sahlqvist fixed point term with the same property.

EXAMPLE 4.9. Let $\mathbb{N}$ be the set of natural numbers and $R=\{(n, n-$ $1),(n, n+1): n>0\}$. Let $\mathcal{A}$ be the algebra of all finite and cofinite subsets of $\mathbb{N}$. For each $U \subseteq \mathbb{N}$ we let $\langle R\rangle U=\{n \in \mathbb{N}: R[n] \cap U \neq \emptyset\}$, where $R[n]=\{m \in \mathbb{N}: R(n, m)\}$. It is well known that $\mathcal{A}$ is a conjugated BAO. It is called a conjugated modal algebra because of the close connection with modal logic, see e.g., [5, Section 5]. It is also well known that the completion $\overline{\mathcal{A}}$ of $\mathcal{A}$ consists of all subsets of $\mathbb{N}$ with $\langle R\rangle$ defined as above. Observe that the only pre-fixed point of the term $\delta=\diamond \diamond x \vee \square \perp$ in $\mathcal{A}$ is the whole set $\mathbb{N}$. So the interpretation of $\mu x \delta$ is equal to $\mathbb{N}=1_{\mathcal{A}}$. However, the only pre-fixed points of $\delta$ in $\overline{\mathcal{A}}$ are $\mathbb{N}$ and the set $E$ of all even numbers. Therefore, the interpretation of $\mu x \delta$ in $\overline{\mathcal{A}}$ is equal to $E$. Finally, note that as $\mu x \delta$ is positive, $\gamma=\neg \mu x \delta$ is a Sahlqvist fixed point term. Thus, from the above we obtain that $\gamma=0$ is valid in $\mathcal{A}$, but $\gamma=0$ is not valid in $\overline{\mathcal{A}}$.

\section{Conclusions and future work}

In this paper we defined Sahlqvist fixed point equations and fixed point BAOs. For each BAO $\mathcal{A}$, we interpreted fixed point operators in a "nonclassical' way relative to some set $\mathcal{F} \subseteq \mathcal{A}$. We proved that for each fixed point $\mathrm{BAO} \mathcal{A}$ and $\mathcal{F} \subseteq \mathcal{A}$, every Sahlqvist fixed point equation valid in $\mathcal{A}$ relative to $\mathcal{F}$ is also valid in the completion $\overline{\mathcal{A}}$ relative to $\mathcal{F}$. We also gave an example of a conjugated BAO $\mathcal{A}$ and Sahlqvist fixed point equation that is not preserved under the completion $\overline{\mathcal{A}}$ provided that the fixed point operators are interpreted in a classical way as meets and joins of all pre and post-fixed points, respectively. We left it as on open problem to find a fixed point BAO with the same property.

The last question is related to the following problem: Given a $\mathrm{BAO} \mathcal{A}$ can we find a fixed point $\mathrm{BAO} \mathcal{B}$ such that $\mathcal{A}$ is a subalgebra of $\mathcal{B}$ and $\mathcal{B}$ is least such? Obviously, the completion $\overline{\mathcal{A}}$ is a fixed point BAO containing $\mathcal{A}$ as a subalgebra. But can we find a 'smaller' one? In other words, can we define fixed point completions of BAOs?

In this paper we consider lower extensions of conjugated operators on a BAO. We could define the upper extension of a map $f$ on a BAO $\mathcal{A}$ by $\widehat{f}(b)=$ $\bigwedge^{\overline{\mathcal{A}}}\{f(c): c \in \mathcal{A}, c \geq b\}$. As follows from [8] (see also [9, Theorem 3.1] and [18, Proposition 3.19]), if $f$ is a unary conjugated operator, then $\bar{f}=\widehat{f}$. If $f$ is not unary though, then $\bar{f}$ and $\widehat{f}$ may differ [8] (see also [18, Example 3.20]). We leave it as an open problem to investigate completions of fixed point BAOs, when we consider upper extensions of operators. 
Finally, we note that the skeleton of a Sahlqvist fixed point term is an $L$ term. Fixed point operators are only allowed in the negative terms substituted in the skeleton. Currently we are unable to extend Lemma 4.5 and the claim of Theorem 4.7 to skeletons that involve fixed point operators (e.g., as in the Sahlqvist fixed point formulas of [3]). We leave it as an (interesting) open problem whether the notions of skeletons or boxed atoms could be generalized so that they involve fixed point operators and so that the analogues of Lemma 4.5 and Theorem 4.7 for these terms still hold.

\section{REFERENCES}

1. R. Balbes and P. Dwinger, Distributive lattices, University of Missouri Press, Columbia, Mo., 1974.

2. J. van Benthem, Modal logic and classical logic, Bibliopolis, Naples, 1985.

3. J. van Benthem, N. Bezhanishvili, and I. Hodkinson, Sahlqvist correspondence for modal mu-calculus, Studia Logica, to appear.

4. N. Bezhanishvili and I. Hodkinson, Sahlqvist theorem for modal fixed point logic, Theoretical Computer Science, to appear.

5. P. Blackburn, M. de Rijke, and Y. Venema, Modal logic, Cambridge University Press, 2001

6. L. L. Esakia, Topological Kripke models, Soviet Math. Dokl. 15 (1974), 147-151.

7. M. Gehrke, J. Harding, and Y. Venema, MacNeille completions and canonical extensions, Trans. Amer. Math. Soc. 358 (2006), no. 2, 573-590.

8. S. Givant and Y. Venema, The preservation of Sahlqvist equations in completions of Boolean algebras with operators, Algebra Universalis 41 (1999), no. 1, 47-84.

9. J. Harding and G. Bezhanishvili, MacNeille completions of modal algebras, Houston J. Math. 33 (2007), no. 2, 355-384.

10. B. Jónsson, On the canonicity of Sahlqvist identities, Studia Logica 53 (1994), no. 4 473-491.

11. B. Jónsson and A. Tarski, Boolean algebras with operators. I, Amer. J. Math. 73 (1951), 891-939.

12. H. M. MacNeille, Partially ordered sets, Trans. Amer. Math. Soc. 42 (1937), no. 3 , 416-460.

13. J. D. Monk, Completions of Boolean algebras with operators, Math. Nachr. 46 (1970), $47-55$.

14. H. Ribeiro, A remark on Boolean algebras with operators, Amer. J. Math. 74 (1952), 163-167.

15. G. Sambin and V. Vaccaro, A new proof of Sahlqvist's theorem on modal definability and completeness, Journal of Symbolic Logic 54 (1989), 992-999.

16. L. Santocanale, Completions of $\mu$-algebras, Annals of Pure and Applied Logic $\mathbf{1 5 4}$ (2008), no. 1, 27-50.

17. R. Sikorski, Boolean algebras, Ergebnisse der Mathematik und ihrer Grenzgebiete, N. F., Heft 25, Springer-Verlag, Berlin, 1960.

18. M. Theunissen and Y. Venema, MacNeille completions of lattice expansions, Algebra Universalis 57 (2007), no. 2, 143-193.

Nick Bezhanishvili: Department of Computing, Imperial College London, South Kensington Campus, London SW7 2AZ, UK, nbezhani@doc.ic.ac.uk. homepage: http://www.doc.ic.ac.uk/ nbezhani/

Ian Hodkinson: Department of Computing, Imperial College London, South Kensington Campus, London SW7 2AZ, UK, imh@doc.ic.ac.uk. homepage: http://www.doc.ic.ac.uk/ imh/ 
